\title{
Comunicação Digital para Matemática ${ }^{1}$
}

\author{
Vinícius Teixeira da Silva ${ }^{2}$ \\ Marcus Vinicius de Azevedo Basso ${ }^{3}$
}

\author{
Universidade Federal do Rio Grande do Sul (UFRGS) \\ Laboratório de Estudos Cognitivos - Instituto de Psicologia - Rua Ramiro Barcelos, 2600 \\ Bairro Santa Cecília - CEP: 90035-003 - sala 1 \\ Instituto de Matemática - Av. Bento Gonçalves, 9500 - Prédio 43111 \\ Bairro Agronomia - CEP 91509-900 \\ Porto Alegre - RS - Brasil \\ jetvini@hotmail.com, mbasso@ufrgs.br
}

\begin{abstract}
Resumo
O Governo Federal Brasileiro tem investido em projetos de educação a distância desde a década de setenta. O ensino superior na área da Matemática é um caso particular e que requer algumas ferramentas para interação aluno/aluno e aluno/professor que até então não existiam. A ausência, nos teclados dos computadores, da maioria dos símbolos utilizados em expressões matemáticas e a falta de um programa que possibilitasse a edição de expressões e comunicação síncrona são obstáculos importantes para a educação matemática a distância. Para contribuir para a solução dessas dificuldades, após um ano de esforços, conseguimos desenvolver um programa que apresenta as seguintes características: permite a edição de textos matemáticos, estabelece a comunicação em tempo síncrono, é compatível com qualquer sistema operacional (portabilidade), oferece segurança aos usuários, tem uma boa usabilidade e exige apenas os conhecimentos básicos de navegação na Internet. Este programa foi desenvolvido na linguagem JAVA, da Sun Computers e, para sua execução, requer apenas a instalação do J2SE Runtime Enviroment.
\end{abstract}

Palavras-chave: comunicação matemática, educação a distância

\begin{abstract}
The Brazilian Federal Government has invested in education projects in the distance since the decade of seventy. Superior education in the area of the Mathematics is a particular case and that it requires some tools for interaction student/student and student/teacher that until then did not exist. The absence, in the keyboards of the computers, the majority of the symbols used in mathematical expressions and the lack of one program that made possible the edition of expressions and synchronous communication are important obstacles for the mathematical education in the distance. To contribute for the solution of these difficulties, after one year of efforts, we obtain to develop a program that presents the following characteristics: it allows the edition of mathematical texts, establishes the communication in real time, is compatible with any operational system, it offers security to the users, it has a good usability and it demands only the basic knowledge of navigation in the Internet. This program was developed in language $J A V A$, of Sun Computers and, for its execution, it requires only the installation of the J2SE Runtime Enviroment.
\end{abstract}

Key-words: mathematics communication, distance learning

\footnotetext{
${ }^{1}$ Esse trabalho está inserido no Projeto Amadis - Um Ambiente Virtual para apoio ao Desenvolvimento de Projetos de Aprendizagem com financiamento do MCT/FINEP

${ }^{2}$ Acadêmico do Curso de Licenciatura de Matemática - Instituto de Matemática - UFRGS.

${ }^{3}$ Professor do Curso de Licenciatura de Matemática - Instituto de Matemática - UFRGS 


\section{Introdução}

A comunicação com o uso da informática evoluiu muito desde a criação desta ciência. O computador como ferramenta multimídia tem transcendido de útil para indispensável para qualquer área do conhecimento, em particular para a Matemática.

Com os atuais incentivos do nosso governo aos projetos de educação a distância é inevitável que nossas universidades cada vez mais precisem de instrumentos para propagar e sustentar seus cursos de em todos os níveis através da Internet.

Porém tentemos utilizar uma sala de bate-papo ou um programa de conversação síncrona como o Ms Messenger para conversar a respeito da resolução de um problema matemático onde se faça necessário apresentar uma expressão com símbolos.

É claro que ao olharmos para um teclado facilmente perceberemos que ali faltam muitos dos símbolos utilizados para construir expressões matemáticas.

Por exemplo, consideremos a seguinte situação: resolver um problema de geometria, nesse caso, uma questão do Concurso Vestibular - UFRGS - 2001, solicitando que seja dada uma justificativa para opção feita via utilização de um formulário na web. (figura 1)

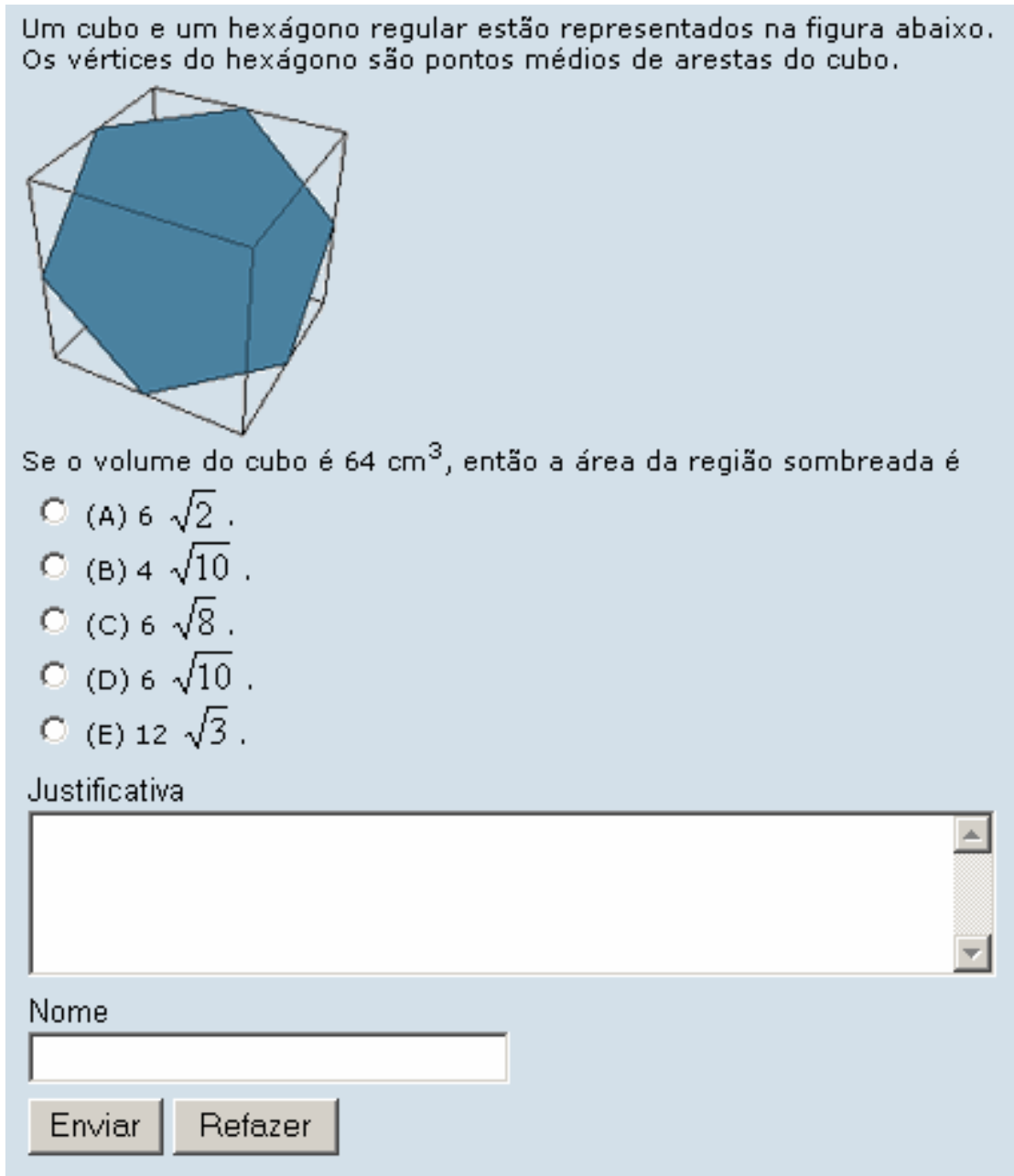

Figura 1: questão de múltipla escolha - formulário web com botões de opção e caixa de texto

Uma primeira alternativa para apresentar tal justificativa seria utilizar apenas a linguagem corrente, descrevendo o procedimento necessário para resolver a questão, como no extrato retirado de uma página da web. (Extrato 1) 
Nome: ------ Data: 20/10/2005 Hora: 11:18:51

Alternativa: $E$

Justificativa: Primeiramente calculamos o lado do cubo, que é 4. Depois, sabendo que cada vértice do hexágono divide o lado do cubo em dois, calculamos o lado do hexágono regular, sabendo que ele é a diagonal de um quadrado de lado 2. Após isso calculamos a área de um triângulo que forma o hexágono e depois multiplicamos por 6 e encontramos a área da região sombreada.

\section{Extrato 1 - justificativa para a escolha da opção}

Já nos extrato 2, a seguir, podemos ver outro exemplo da limitação imposta pela linguagem disponível para escrever tal justificativa. Nesse caso, uma primeira pergunta que os estudantes fazem é: "Como eu escrevo a raiz quadrada?". Essa ausência impõe o uso da própria expressão "raiz quadrada" ou o que é comumente utilizado em aplicativos de Matemática, SQRT (parâmetro). Mesmo expressões mais simples, como a que envolve a utilização de uma potência ou um índice, e que para ser resolvido têmse que apelar para o uso de código HTML, tornam o trabalho mais difícil e desviam o foco do próprio trabalho que é o de pensar na solução para a questão. (extratos 3 e 4)

Nome: ------ Data: 20/10/2005 Hora: 11:26:45

Alternativa: $E$

Justificativa: Se o volume do cubo é $64 \mathrm{~cm}^{3}$ então cada aresta mede $4 \mathrm{~cm}$ a aresta do cubo dividida ao meio mede $2 \mathrm{~cm}$, então para calcularmos o tamanho do lado do hexágono usamos um triângulo retângulo que tem catetos medindo $2 \mathrm{~cm}$ e hip igual ao tamanho do lado do hexágono. Resolvendo isso encontramos hip= raiz quadrada de 8. Sabendo o tamanho do lado de um hexágono conseguimos determinar a altura dos triângulos que formam o hexágono, altura = raiz quadrada de 6. Logo cada triângulo tem área igual a $2 *$ raiz quadrada de 3 , como são 6 triângulos que formam a área sombreada $A$, então $A=12 *$ raiz quadrada de 3

\section{Extrato 2 - justificativa para a escolha da opção}

Nome: ------ Data: 20/10/2005 Hora: 11:19:34

Alternativa: $E$

Justificativa: Se o volume V do cubo é $64 \mathrm{~cm} 3$, então, se a é a aresta do cubo:

$V=64=>a 3=43=>a=4 \mathrm{~cm}$

Se l é o lado do hexágono, então:

$l 2=(a / 2) 2+(a / 2) 2=2[(a / 2) 2]=2[(4 / 2) 2]=2.22=8=>($ como $l>0) l=2 \operatorname{SQRT}(2)$

Temos que a área A do hexágono é:

$A=6 .[l .(l S Q R T(3) / 2) / 2]=3[\ln S Q R T(3) / 2]=3.8 . S Q R T(3) / 2=24 . S Q R T(3) / 2=$ $12 \operatorname{SQRT}(3)$

\section{Extrato 3 - justificativa para a escolha da opção}

Se o volume $\mathrm{V}$ do cubo é $64 \mathrm{~cm}<\sup >3</$ sup $>$, então, se a é a aresta do cubo: $<$ br $>$

$\mathrm{V}=64=>\mathrm{a}<\sup >3</$ sup $>=4<\sup >3</$ sup $>=>\mathrm{a}=4 \mathrm{~cm}<$ br $>$

Se 1 é o lado do hexágono, então: $<$ br $>$

$1<\sup >2</$ sup $>=(\mathrm{a} / 2)<\sup >2</$ sup $>+(\mathrm{a} / 2)<\sup >2</$ sup $>=2[(\mathrm{a} / 2)<\sup >2</$ sup $>]=$ $2[(4 / 2)<\sup >2</$ sup $>]=2.2<\sup >2</$ sup $>=8=>($ como l $>0) 1=2 \operatorname{SQRT}(2)<$ br $>$

Temos que a área $A$ do hexágono é: $<$ br $>$

$\mathrm{A}=6 .[1 .(1 \mathrm{SQRT}(3) / 2) / 2]=3[1<\sup >2</ \sup >\operatorname{SQRT}(3) / 2]=3.8 . \mathrm{SQRT}(3) / 2=$ 24.SQRT(3)/2 = 12SQRT(3)

\section{Extrato 4 - parte do código html do extrato 3}


As questões acima nos remetem a pensarmos sobre quais são as carências na educação matemática a distância.

Aproveitando as experiências, tanto a de aluno, em formação inicial para professor, quanto a de professor de Matemática, destacamos que freqüentemente precisamos discutir sobre algum problema com colegas e a comunicação é dificultada pela falta de recursos físicos, isto é, a ausência de muitos dos símbolos próprios da área e também pela quantidade de conhecimentos da informática que os agentes de uma interação a distância precisam dominar. Esses agentes têm que demonstrar habilidade em utilizar um conjunto de programas com finalidades distintas (editores de texto, editores de fórmulas, mailers, etc).

O objetivo do projeto é tentar solucionar estes problemas impostos à Educação Matemática a Distância. Para isso, desenvolvemos e continuamos aperfeiçoando uma ferramenta para comunicação digital que deverá unificar as funções dos programas anteriores. Tal software chama-se EMOL - Editor para Matemática On Line.

\section{Material e Métodos aplicados para a produção da ferramenta}

A partir da orientação dos coordenadores do projeto e das constantes discussões sobre a facilitação do atendimento extra-classe com recursos computacionais ficou estabelecida como meta a produção de um programa inovador, que pudesse dar conta das demandas já relatadas.

Uma vez que nosso trabalho centrava-se essencialmente na implementação de um programa, desenvolvemos o objeto da pesquisa nos laboratórios de Ensino a Distância do Instituto de Matemática da UFRGS e de Estudos Cognitivos do Instituto de Psicologia da mesma instituição.

A adequação dos recursos e instalações disponibilizadas torna-se evidente no fato de termos conseguido produzir o que fora proposto.

O material bibliográfico utilizado durante todo processo de desenvolvimento foi indispensável, dada a complexidade do que estava desenvolvendo.

\section{Resultados e Discussão}

Submetemos cinco colegas ao seguinte teste: escrever um texto introdutório em um editor de textos, escrever uma expressão matemática, inseri-la no texto e salvar o resultado. Em seguida escolher um meio para enviar um e-mail para alguém anexando o arquivo produzido no primeiro passo. Os tempos foram cronometrados e obtivemos a média aritmética dos tempos: quatro minutos e quarenta e um segundos.

Então, passamos a segunda etapa do teste: escrever a mesma expressão utilizando o EMOL (nosso programa). A média obtida foi de quarenta segundos. Mesmo sendo uma expressão simples, o fato de necessitar de outro programa para gerála foi um fator importante para a diferença tão grande de médias de tempos entre o que já existe em termos de softwares e o EMOL.

Eis a expressão:

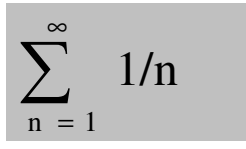

A seguir veremos as telas capturadas para cada etapa do teste. (figura 2, 3, 4 e 5) $\mathrm{Na}$ primeira seqüência de imagens, poderemos contar a utilização do editor de textos, 
um plugin (próprio para gerar expressões matemáticas) e o browser exibindo um webmail.

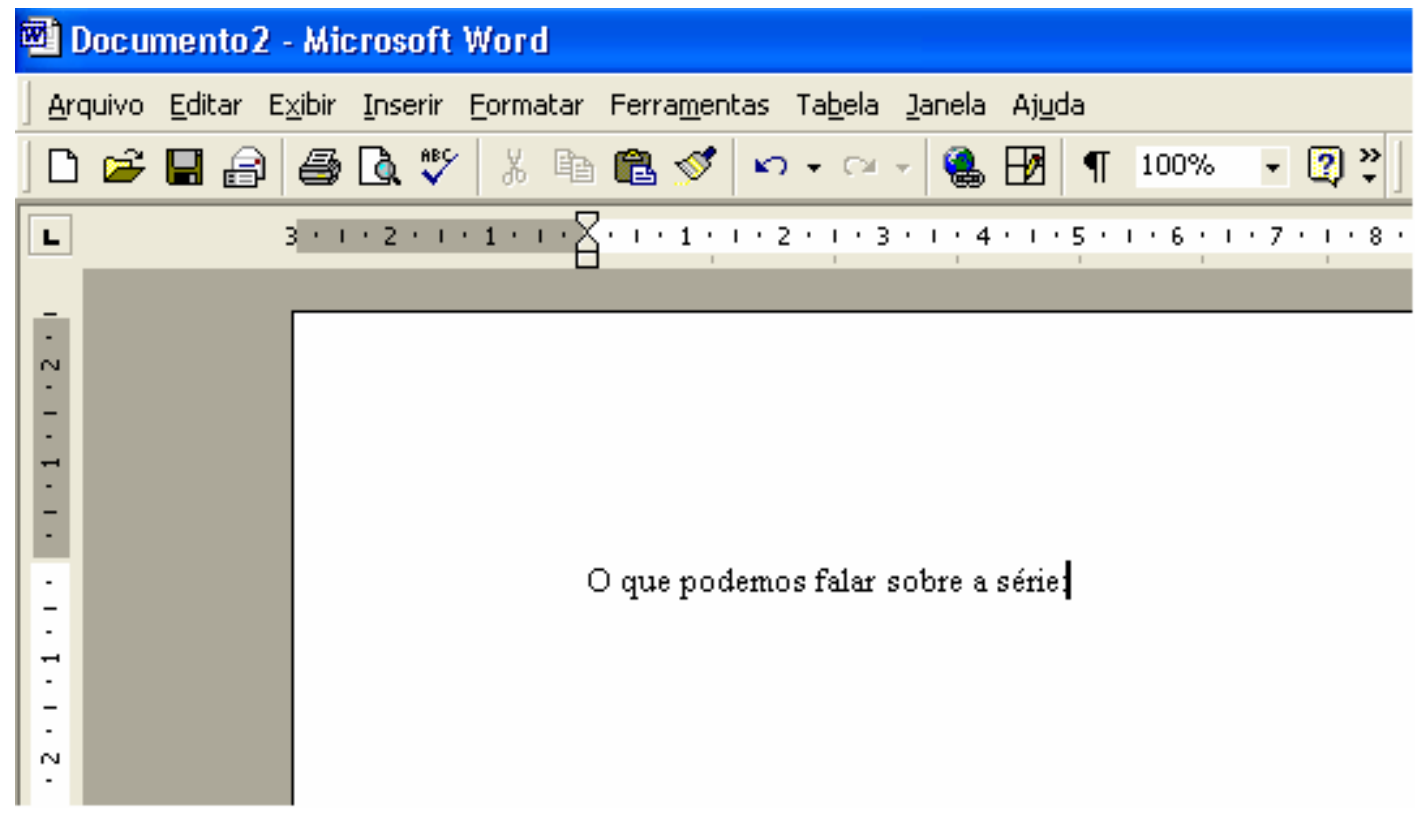

Figura 2 - Produzindo o texto

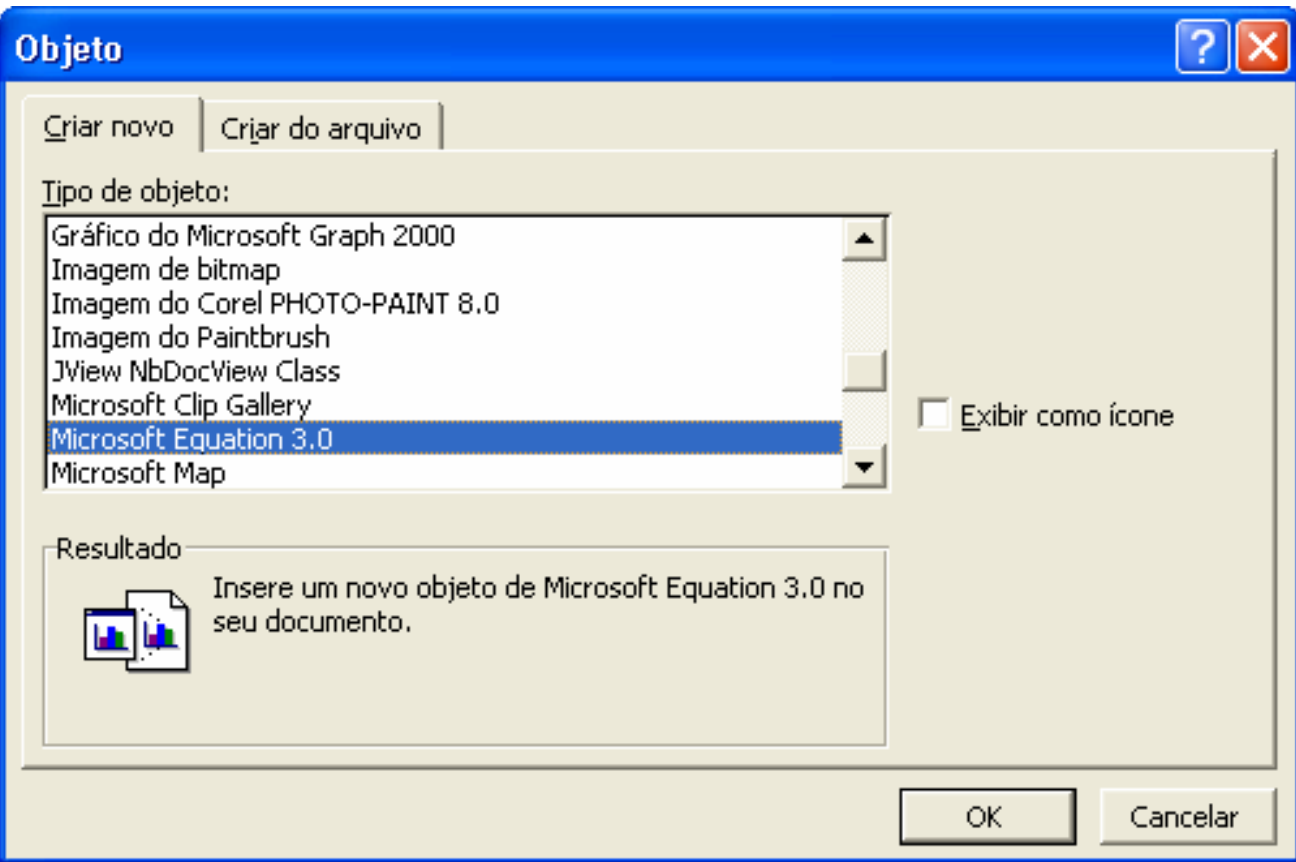

Figura 3 - Inserindo um objeto a partir de um plugin 


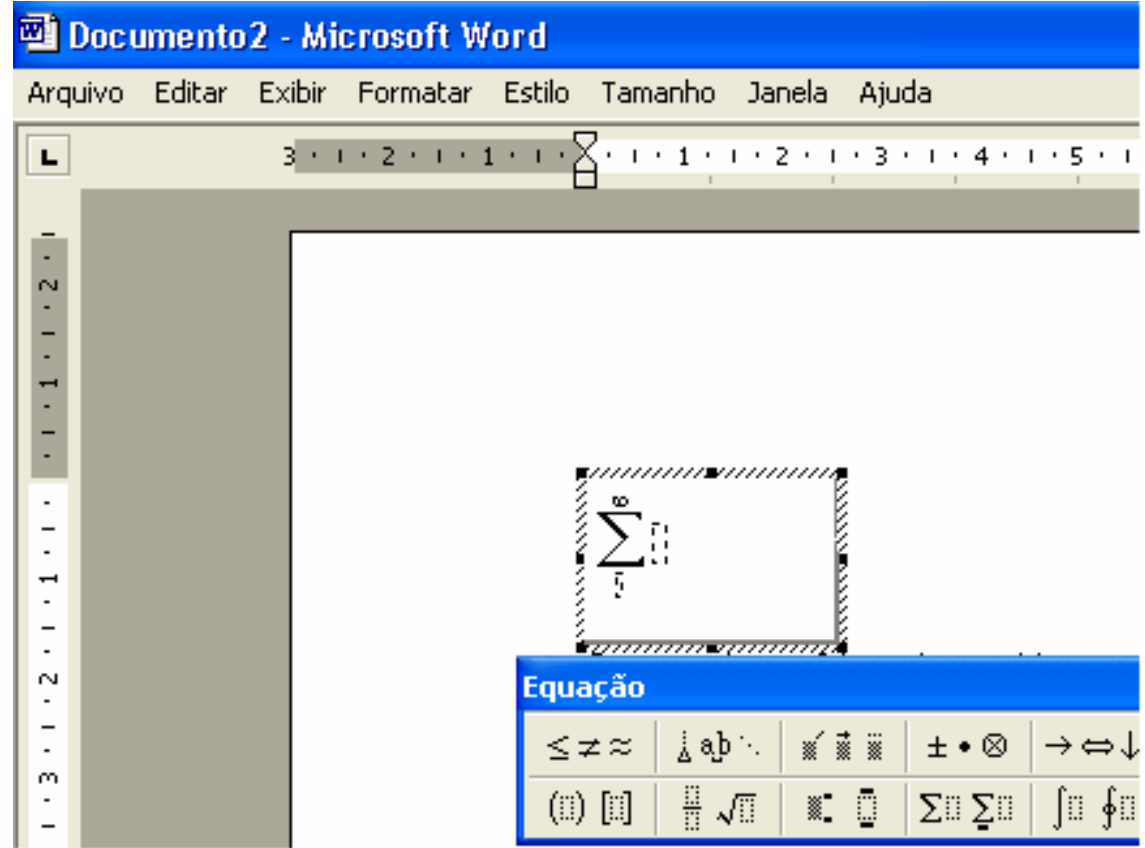

Figura 4 - A edição da expressão matemática

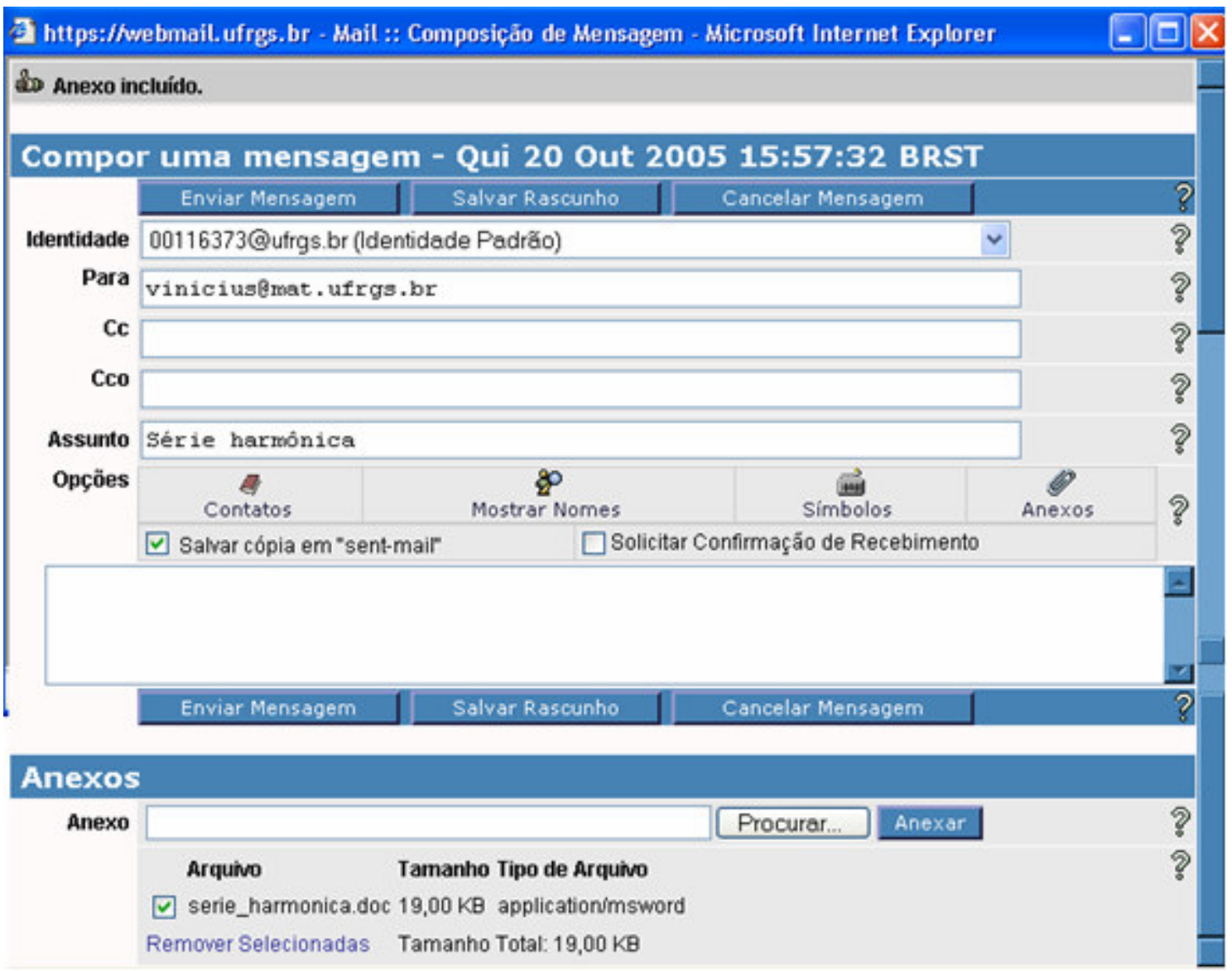

Figura 5 - $O$ arquivo produzido sendo anexado a uma mensagem de correio eletrônico.

No caso da primeira etapa do teste, mesmo tentando criativamente contornar as dificuldades de comunicação, o máximo que se conseguiu foi uma forma assíncrona, isto é, os agentes da interação não estão necessariamente conversando em tempo síncrono e, pode ser que a mensagem venha a ser lida em um outro dia. 
$\mathrm{Na}$ segunda etapa, utilizando o EMOL, os usuários puderam experimentar a produção do texto, da expressão e ainda a comunicação síncrona, conforme o que podemos verificar na figura 6 :

\section{S HTML Test Page - Microsoft Internet Explorer}

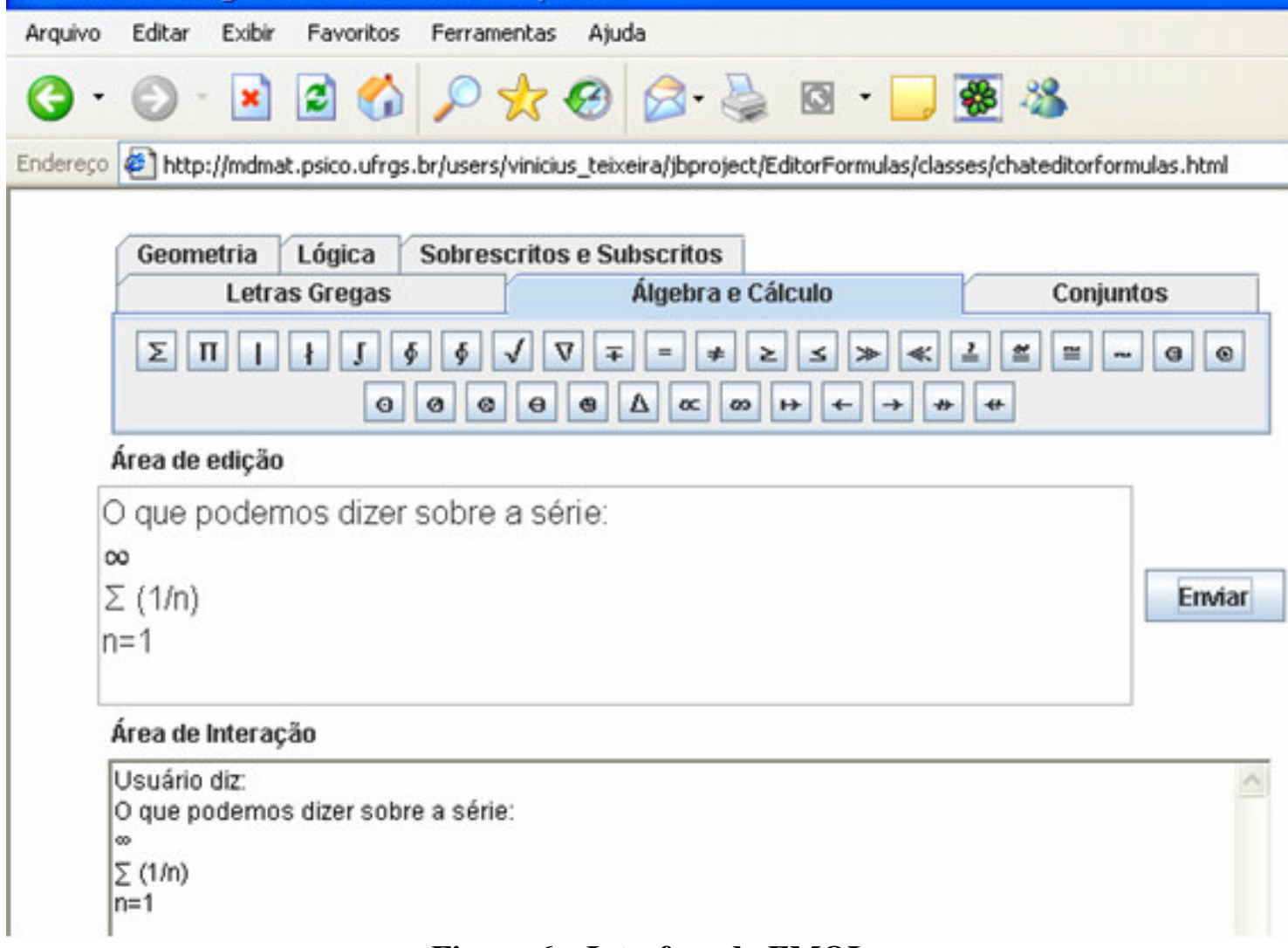

Figura 6 - Interface do EMOL

Nas tabelas 1 e 2, apresentamos uma comparação entre os tempos utilizados para gerar e enviar uma determinada expressão matemática utilizando as conexões ADSL e discada.

\begin{tabular}{|l|l|l|}
\hline$n^{2} a_{n}=\frac{15 n^{3}+n^{2} \sqrt{n^{2}-1}}{5 n^{3}+2 n \sqrt{n+1}-17} \rightarrow \frac{15}{5}=3$ & Ms Equation 3.0/Webmail & $9 \mathrm{~min} 17 \mathrm{~s}$ \\
\hline$n^{2} a_{n}=\frac{15 n^{3}+n^{2} \sqrt{n^{2}-1}}{5 n^{3}+2 n \sqrt{n+1}-17} \rightarrow \frac{15}{5}=3$ & Editor & $2 \mathrm{~min} 58 \mathrm{~s}$ \\
\hline $\begin{array}{l}\text { Infimo de } \mathrm{C}: \text { a) } \mathrm{s} \leq \mathrm{c} \forall \mathrm{c} \in \mathrm{C} ; \\
\text { b) dado qualquer } \varepsilon>0 \exists \mathrm{c} \in \mathrm{C} \text { tal que } \mathrm{c}<\mathrm{s}+\varepsilon\end{array}$ & Ms Word 2003/Outlook & $7 \mathrm{~min}$ \\
\hline $\begin{array}{l}\text { Infimo de } \mathrm{C}: \text { a) } \mathrm{s} \leq \mathrm{c} \forall \mathrm{c} \in \mathrm{C} ; \\
\text { b) dado qualquer } \varepsilon>0 \exists \mathrm{c} \in \mathrm{C} \text { tal que } \mathrm{c}<\mathrm{s}+\varepsilon\end{array}$ & Editor & $1 \mathrm{~min} 52 \mathrm{~s}$ \\
\hline
\end{tabular}

Tabela 1 - tempo despedido na edição/envio de expressão matemática - conexão ADSL 


\begin{tabular}{|l|l|l|}
\hline$n^{2} a_{n}=\frac{15 n^{3}+n^{2} \sqrt{n^{2}-1}}{5 n^{3}+2 n \sqrt{n+1}-17} \rightarrow \frac{15}{5}=3$ & Ms Equation 3.0/Webmail & $12 \mathrm{~min} 38 \mathrm{~s}$ \\
\hline$n^{2} a_{n}=\frac{15 n^{3}+n^{2} \sqrt{n^{2}-1}}{5 n^{3}+2 n \sqrt{n+1}-17} \rightarrow \frac{15}{5}=3$ & Editor & $2 \mathrm{~min} 58 \mathrm{~s}$ \\
\hline $\begin{array}{l}\text { Ínfimo de C: a) } \mathrm{s} \leq \mathrm{c} \forall \mathrm{c} \in \mathrm{C} ; \\
\text { b) dado qualquer } \varepsilon>0 \exists \mathrm{c} \in \mathrm{C} \text { tal que } \mathrm{c}<\mathrm{s}+\varepsilon\end{array}$ & Ms Word 2003/Outlook & $9 \mathrm{~min} 6 \mathrm{~s}$ \\
\hline $\begin{array}{l}\text { Infimo de } \mathrm{C}: \text { a) } \mathrm{s} \leq \mathrm{c} \forall \mathrm{c} \in \mathrm{C} \text {; } \\
\text { b) dado qualquer } \varepsilon>0 \exists \mathrm{c} \in \mathrm{C} \text { tal que } \mathrm{c}<\mathrm{s}+\varepsilon\end{array}$ & Editor & $1 \mathrm{~min} 52 \mathrm{~s}$ \\
\hline
\end{tabular}

Tabela 2 - tempo despedido na edição/envio de expressão matemática - conexão discada

\section{Conclusões}

Utilizando o EMOL, quais as conseqüências do que foi exposto para a educação matemática a distância? Primeiramente, o aluno/professor não precisará ter conhecimentos sobre diversos programas. Isto significa poupar tempo em treinamento de profissionais nas universidades, na dispensabilidade de inserir forçadamente hábitos nos alunos e professores de uma área que não são da informática.

Outra vantagem de não produzir expressões matemáticas em softwares diversos é a garantia de que não haverá incompatibilidade de leitura de arquivos nos computadores dos agentes da interação.

Incompatibilidades geradas pelo uso de softwares diferentes ou de versões diferentes de um mesmo software.

Tudo isto tem sido altamente excludente, ou seja, um agente determina qual software será utilizado e os outros devem tentar instalar o programa nos seus computadores. Mas todos possuem computadores capazes de rodar qualquer software? Um último obstáculo que vemos sendo transposto é o tempo. Escrever uma fórmula, o passo a passo de uma derivação de função ou a explicação de um conceito de análise requer um tempo. Enviar o que foi produzido e esperar o retorno é óbvio que requer mais um tempo que não podemos desprezar. Aliás, é o atraso no envio e recebimento das informações um dos principais motivos do desinteresse do aluno em obter ajuda a distância. Com o EMOL e o conceito de comunicação síncrona que este proporciona, vemos um melhor aproveitamento do tempo, explorando todas as possibilidades de sanar dúvidas na medida em que se esteja desenrolando um diálogo matemático.

Este programa foi desenvolvido sob a forma de applet. Uma applet é um miniaplicativo que roda dentro de uma página Web. Ele é rodado na JVM - Java Virtual Machine, que realmente é uma máquina virtual que fica dentro do computador no qual ela está rodando. Assim nosso programa não tem contato com o computador real; ele conhece apenas a máquina virtual. A principal implicação disso é impedir que os usuários que estejam conversando através da nossa ferramenta tenham acesso às informações salvas nos computadores dos outros usuários e que tenham seguras também suas informações.

A única exigência em termos de instalação de software é que o usuário tenha em seu computador o JRE 1.5, que é um pacote livre distribuído pela Sun Computers para poder rodar os miniaplicativos Java no computador. Com isto, basta o usuário a partir do seu browser acessar o endereço da página onde está o Editor-Mensageiro. 
A tecnologia utilizada para desenvolver o programa foi a linguagem de programação Java. Para facilitar a construção visual e a orientação aos objetos utilizamos o Borland JBuilder 2005 Foudation.

Nossa applet não utiliza Mathml, ele cria textos com a possibilidade de inserir caracteres Unicode.

O funcionamento é bem simples: o usuário acessa uma página onde uma primeira applet armazena seu nome ou nickname em um servidor de chat (que faz parte do nosso projeto) e utiliza uma segunda applet - um cliente de chat - para interagir com os presentes. É neste cliente de chat que o usuário experimenta a facilidade de escrever textos e expressões matemáticas.

Após enviar o que fora escrito em um campo de texto, a mensagem é processada pelo servidor e ecoada para todos os clientes.

O programa está em fase de finalização e estará disponível na rede para uso geral a partir da segunda quinzena de novembro.

Ainda temos perspectivas de melhoras para versões posteriores, como a possibilidade de trabalhar com imagens. Além disso, o caráter open source que desejamos atribuir ao nosso software irá permitir contribuições de outros programadores para sua melhora e a possível implementação do mesmo para o desenvolvimento de ferramentas para outras áreas do conhecimento como a Química, a Física ou outras, todas com suas simbologias.

\section{Referências}

Thomas, Michael D. (1997) Programando em JAVA para a Internet. São Paulo: Makron Books.

Deitel, Harvey M. (2003) JAVA: Como programar. 6. Ed. São Paulo: Pearson Prentice Hall.

Wood, A. (1999) Unicode and Multilingual Support in HTML, Fonts, Web Browsers and Other Applications. Disponível em: <http://www.alanwood.net/ unicode/\#links>. Acesso em 15 mar. 2005. 